\title{
Numerical Filtering for the Operation of Robotic Manipulators through Kinematically Singular Configurations
}

\author{
Anthony A. Maciejewski \\ School of Electrical Engineering \\ Purdue University \\ West Lafayette, Indiana 47907 \\ Charles A. Klein* \\ Department of Electrical Engineering \\ Ohio State University \\ Columbus, Ohio 43210 \\ Received December 21, 1987; accepted June 6, 1988
}

The loss of independent degrees of freedom at singular configurations is an inherent characteristic of robotic manipulators. Due to the unavoidable singularity of mechanical wrists, singular configurations cannot be avoided by simply restricting the bounds of the workspace. Techniques for operating at singular configurations without inducing unacceptably high joint velocities or end effector tracking errors are presented. Extensions to the damped least-squares formulation which incorporate estimates of the proximity to singularities and selective filtering of singular components are illustrated. The generality of the technique presented is illustrated in a computer simulation of a commercially available manipulator operating through singular configurations.

特頼点において自由度の独立性が失われることは、ロボットマニピュレータに付

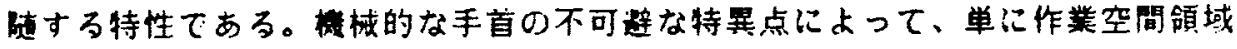

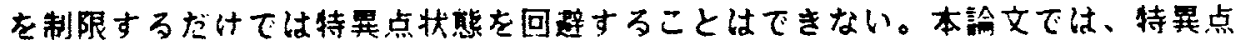
状㷱においても、受け入れがたい高い開節速度やエンドイフェクタのトラッキン クエラーを生ずることなく聚䡃できる手法が述べられる。特暴点への接近度の評 価を組み込んた减変最小自乗形式への拉張と、特晎值成分の㟟抧的フィルタが示

*The work was performed at the Department of Electrical Engineering, The Ohio State University, 2015 Neil Avenue, Columbus, $\mathrm{OH}, 43210$. This work was supported by the National Science Foundation under grant DMC-8421321.

Journal of Robotic Systems, 5(6), 527-552 (1988)

(C) 1988 by John Wiley \& Sons, Inc.

CCC 0741-223/88/060527-26\$4.00 
される。さらにここで述べられた手法の一般性は、特㟟点を通る動作をする市販

マニビュレータのコンビュータシミュレーションにより示される。

\section{INTRODUCTION}

All articulated manipulators, with the exception of some used only for positioning, can be shown to possess singular configurations which limit the effective number of independent degrees of freedom. ${ }^{3}$ These singular configurations restrict the motion of the end effector and can induce high joint velocities and spurious motions. Due to these undesirable characteristics there has been a significant amount of effort aimed at avoiding the operation of a manipulator at or near a singular configuration. ${ }^{2-7}$ Unfortunately, due to the inevitable singularity of pointing or orienting wrists, singular configurations cannot be restricted to isolated regions of the workspace.

The effects of singularities are frequently presented with respect to the resolved motion rate control formulation ${ }^{8}$ given by

$$
\dot{\mathbf{x}}=\boldsymbol{J} \dot{\boldsymbol{\theta}}
$$

where $\dot{\boldsymbol{x}}$ represents the commanded end effector velocity, $\dot{\boldsymbol{\theta}}$ represents the joint angle velocities and $J$ is the Jacobian matrix. In this form singularities can be identified by a mathematical change in rank of $J$ which physically represents the inability of the manipulator to achieve an arbitrary end effector velocity. Since inverses of rank deficient matrices are undefined, the pseudoinverse has been proposed as a means of obtaining solutions to (1). ${ }^{8}$ Unfortunately, pseudoinverse solutions have an undesirable discontinuity for arbitrarily small perturbations which result in a change of rank. ${ }^{9}$ The difficulty, therefore, is not at singularities but the ill-conditioned transition between singular and nonsingular configurations which results in oscillations and unacceptably high joint velocities. It is important to note that these difficulties are not unique to the resolved rate formulation but are an inherent part of the transformation between Cartesian and joint spaces.

One approach to achieving the desired continuity between singular and nonsingular configurations, independently proposed in Refs 10 and 11 , is to use a well-conditioned formulation based on weighting the accuracy of tracking the end effector velocity with the norm of the joint angle velocity, a method sometimes referred to as damped least squares. The success of this approach is based on varying the weighting factor between these two criteria so that unnecessary end effector tracking errors are not produced in wellconditioned configurations. The loss of an independent degree of freedom at a singularity, however, also need not adversely affect end effector tracking. Clearly, if the commanded end effector velocity does not include the unachievable component, then there is no physical reason for inducing errors into the well-conditioned components. The ability to identify such cases requires further information about the manipulator configuration and the commanded end effector velocity.

The approach presented here is to extend the concept of damped leastsquares solutions by incorporating a technique for determining the minimum 
singular value and the directions associated with the lost degrees of freedom. This allows a more accurate means of determining proximity to singularities which can then be used to set an appropriate weighting factor. In addition, knowledge of the direction of any lost degrees of freedom permits the use of a formulation for selectively filtering out individual components from the commanded end effector velocity. This formulation more accurately reflects the physical constraints on the manipulator so that performance can match kinematic capabilities.

\section{PROPERTIES OF DAMPED LEAST-SQUARES SOLUTIONS}

The concept of considering a solution's norm along with the accuracy to which it solves a set of linear equations was first proposed by Levenberg in Ref. 12 where he coined the phrase "damped least squares". Since that time the use of damped least squares, also referred to as regularization, has become one of a number of common techniques for obtaining solutions to ill-conditioned equations such as those encountered in applications involving measurement or sensing. ${ }^{13,14}$ In terms of the inverse kinematics problem specified by (1) the damped least-squares criterion requires a solution which minimizes the sum

$$
\|\dot{\mathbf{x}}-\boldsymbol{J} \dot{\boldsymbol{\theta}}\|^{2}+\lambda^{2}\|\dot{\boldsymbol{\theta}}\|^{2}
$$

where $\lambda$ is a weighting factor, sometimes referred to as the damping factor, which can be used to set the relative importance of the minimum residual criterion (i.e., end effector tracking error) versus the norm of the solution. The solution which satisfies this criterion for a particular value of $\lambda$ will be denoted as $\dot{\theta}^{(\lambda)}$. The damped least-squares criterion results in the augmented system of equations

$$
\left[\begin{array}{c}
J \\
\lambda I
\end{array}\right] \dot{\boldsymbol{\theta}}=\left[\begin{array}{l}
\dot{\mathbf{x}} \\
0
\end{array}\right]
$$

where the solution can be obtained by solving the consistent set of equations

$$
\left(J^{T} J+\lambda^{2} I\right) \dot{\theta}=J^{T} \dot{x}
$$

which results in $\dot{\theta}^{(\lambda)}$. It is easily shown ${ }^{15}$ that this $\dot{\theta}^{(\lambda)}$ represents the solution with the minimal residual over all $\dot{\theta}$ whose norm does not exceed that of $\dot{\theta}^{(\lambda)}$.

The properties of the damped least-squares solution are perhaps best revealed by the use of the singular value decomposition (SVD). The SVD of the Jacobian $J$, order $m \times n$, can be represented in the form

$$
J=\sum_{i=1}^{\min (m, n)} \sigma_{i} \mathbf{u}_{i} \mathbf{v}_{\mathbf{i}}^{\mathbf{T}}
$$

where $v_{1}$ and $u_{4}$ denote the input and output singular vectors and the singular values, denoted by $\sigma_{i}$, are typically ordered from largest to smallest so that

$$
\sigma_{1} \geq \sigma_{2} \geq \sigma_{\min (m, n)} \geq 0
$$


where the number of nonzero singular values is the rank of $J$. The matrix $J$ is allowed to be non-square to allow for kinematically redundant manipulators. The minimum singular value of $J$ has a special significance since it is the only accurate measure of proximity to singularities. Physically it represents the ratio of end effector velocity to joint velocity in the direction for which it is most difficult to move. This direction is given by the associated output singular vector.

By writing $\dot{x}$ in terms of the basis specified by the output singular vectors

$$
\dot{x}_{i}=\mathbf{u}_{i}^{\mathbf{T}} \dot{x}
$$

the components of the damped least-squares solution can be written as

$$
\dot{\boldsymbol{\theta}}_{i}^{(\lambda)}=\frac{\sigma_{i}}{\sigma_{i}^{2}+\lambda^{2}} \mathbf{v}_{i} \dot{x}_{i}
$$

It is important to note that inclusion of the vector norm criterion into the least-squares process only affects the magnitude of the singular values; the singular vectors remain unchanged. From (8) one can see that for $\sigma_{i}$ much larger than $\lambda$ the damped least-squares solution has little effect since

$$
\frac{\sigma_{i}}{\sigma_{i}^{2}+\lambda^{2}} \approx \frac{1}{\sigma_{i}}
$$

which results in approximately the same value as the standard least squares. However for $\sigma_{i}$ that are on the order of $\lambda$, the $\lambda$ term in the denominator "damps" the potentially high norm of that component of the solution so that

$$
\max \left(\left\|\dot{\boldsymbol{\theta}}_{i}^{(\lambda)}\right\|\right)=\frac{\dot{x}_{i}}{2 \lambda}
$$

which occurs when $\sigma_{i}=\lambda$. For $\sigma_{i}$ smaller than $\lambda, \dot{\theta}_{i}^{(\lambda)}$ approaches zero as $\sigma_{i}$ approaches zero which demonstrates the desired continuity in the solution in spite of the change in the rank of $J$. A plot of the norm of a component of the damped least-squares solution as compared to the standard least squares as a function of $\sigma_{i}$ is presented in Figure 1.

The norm of the complete solution is obtained by combining each of the components from (8) which results in

$$
\left\|\dot{\theta}^{(\lambda)}\right\|^{2}=\sum_{i=1}^{r} \dot{x}_{i}^{2}\left[\frac{\sigma_{i}}{\sigma_{i}^{2}+\lambda^{2}}\right]^{2}
$$

where $r$ is the rank of $J$. The above equation clearly demonstrates that for positive $\lambda$ the norm is monotonically decreasing and approaches zero as $\lambda$ approaches infinity. This decrease in norm, however, is unavoidably ac- 


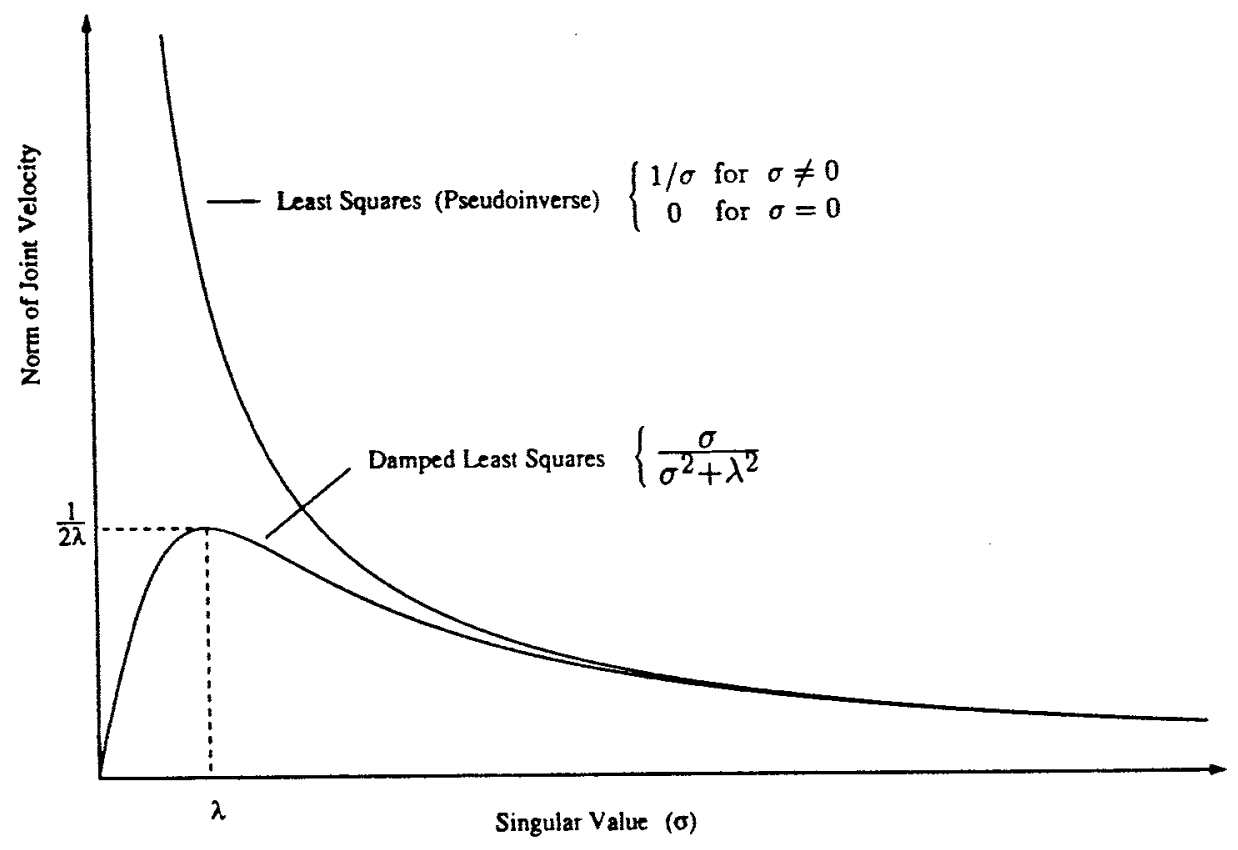

Figure 1. A comparison of the damped and undamped least-squares solutions as a function of the singular value showing the norm of the joint velocity for a component in the direction of the singular vector associated with the plotted singular value.

companied by an increase in the resultant residual. The residual, as a function of $\lambda$, is given by

$$
\left\|\dot{\mathbf{x}}-\boldsymbol{J} \dot{\boldsymbol{\theta}}^{(\lambda)}\right\|^{2}=\sum_{i=1}^{r} \dot{x}_{i}^{2}\left[\frac{\lambda^{2}}{\sigma_{i}^{2}+\lambda^{2}}\right]^{2}+\sum_{i=r+1}^{m} \dot{x}_{i}^{2}
$$

The second term of the residual represents that portion of $\dot{x}$ which is outside of the range space of $J$ and is therefore not a function of $\lambda$. The first term illustrates how the damping factor $\lambda$ affects the resultant residual, the magnitude of which is a monotonically increasing function with a minimum at $\lambda=0$.

The characteristics of the damped least-squares solution are similar to those obtained using the truncated SVD. ${ }^{13}$ The truncated SVD solution of a system of linear equations described by (1), denoted here by $\dot{\theta}^{(k)}$, is defined as

$$
\dot{\theta}^{(k)}=\sum_{i=1}^{k} \frac{1}{\sigma_{i}} \mathbf{v}_{i} \mathbf{u}_{i}^{\mathbf{T}} \dot{\mathbf{x}}
$$

where $k$ is an integer less than or equal to the rank $r$. The truncated SVD reduces the solution norm by removing all components of the solution which correspond to small singular values while retaining all of those associated with 
larger singular values. The parameter $k$ is used to define small and large such that $\sigma_{i}$ for $i \leq k$ are large and $\sigma_{i}$ for $i>k$ are considered small. It can be shown that $\dot{\boldsymbol{\theta}}^{(k)}$ is the minimum residual solution for all $\dot{\boldsymbol{\theta}}$ in the $\boldsymbol{k}$-dimensional subspace spanned by $v_{1}$ for $i \leq k .{ }^{15}$ For cases where $\sigma_{k} \gg \sigma_{k+1}$ such that there is a large gap between the large and small singular values and $\lambda$ is chosen to be midway between $\sigma_{k}$ and $\sigma_{k+1}$, the results for the two types of solutions will be approximately the same.

\section{DETERMINING AN APPROPRIATE DAMPING FACTOR}

One of the difficulties in successfully applying the damped least-squares formulation is determining the optimum value for the damping factor $\lambda$. If there exist hard constraints on the maximum allowable joint velocity or end effector tracking error then an iterative technique based on the monotonic behavior of these functions can be applied. Unfortunately, this technique requires repeated solution of (4) which is computationally undesirable, particularly with respect to real-time control. If the SVD of $J$ is available then Newton's method can be applied to the nonlinear equations (11) or (12) to solve for an optimum damping factor.$^{16}$ Once again, however, the SVD is in general too computationally expensive for use in real-time control.

A usable approach to dynamically setting the damping factor is to vary it as a function of the manipulability. ${ }^{11}$ The manipulability, defined as the square root of the determinant of $J J^{\dot{T} 17}$ is easy to compute and has zeros which coincide with the singularities of $J$. Unfortunately, the manipulability measure cannot guarantee an accurate estimate of proximity to singularities, the only reliable measure of this quantity being the minimum singular value. ${ }^{18}$ Since the manipulability measure is the product-of the singular values, it can be held constant for an arbitrarily large variation in both the condition number and the minimum singular value. The discussion in Ref. 10 with regards to choosing an appropriate damping factor centers around the condition number of the matrix $\left(J^{T} J+\lambda^{2} I\right)$. A bound on this quantity prevents ill-conditioned problems in which solutions are suspect due to the amplification of unavoidable roundoff errors present in numerical calculations. A technique for computing an estimate of this condition number is given in Ref. 19, however, it provides only a lower bound and is applicable for only those manipulators which possess spherical wrist joints.

The approach proposed in this work is to use a damping factor based on an estimate of the minimum singular value. The justification for this choice is due to the fact that bounds on the norms of both the joint angle velocity and the end effector tracking error can be specified solely on the basis of the minimum singular value. In addition, the condition number of the Jacobian is bounded by the inverse of the minimum singular value if $J$ has been normalized so that $\sigma_{1}$ is equal to one. Therefore, by knowing the minimum singular value of $J$, a value for the damping factor can be specified which is guaranteed to satisfy a given constraint on either the joint angle velocity, end effector tracking error, or conditioning of the equations. 
For a constraint on the maximum joint angle velocity over all unit norm commanded end effector velocities, denoted by $\dot{\theta}_{\max }$, a damping factor based on (10) can be specified without even considering the minimum singular value. This value of $\lambda$, however, will be much too conservative for those cases where the minimum singular value is greater than the damping factor, which can be seen from Figure 1. In these cases, a better choice for $\lambda$, which is based on the minimum singular value and is still guaranteed to satisfy the joint velocity constraint, can be obtained from (11) which results in

$$
\lambda\left(\dot{\theta}_{\max }\right)=\sqrt{\frac{\sigma_{\min }}{\dot{\theta}_{\max }}-\sigma_{\min }^{2}}
$$

for those cases where $\sigma_{\min }>\lambda$. Clearly if $\sigma_{\min }>1 / \dot{\theta}_{\max }$, then use 0 for $\lambda$.

The end effector tracking error introduced by using the damped leastsquares formulation is given by the first term of (12). Let $\Delta R$ denote this error relative to the commanded velocity, $\dot{\mathbf{x}}$, so that

$$
\Delta R \leq \frac{\lambda^{2}}{\sigma_{r}^{2}+\lambda^{2}}
$$

Therefore, to stay with a maximum specified value, $\Delta R_{\max }$, the required damping factor can be obtained from

$$
\lambda\left(\Delta R_{\max }\right)=\sqrt{\frac{\sigma_{r}^{2} \Delta R_{\max }}{1-\Delta R_{\max }} .}
$$

Note that this is not the total end effector tracking error but only that introduced by using the damped least-squares solution. If $r$ is not equal to $m$ then the end effector velocity component in the space spanned by $\mathbf{u}_{i}$ for $i=r+1$ to $m$ must be included. This corresponds to the second term in (12) which is the null space of the transformation.

The numerical conditioning of the implicit inversion involved in the damped least-squares solution can be determined by the condition number of the $n$ by $n$ matrix $\left(J^{T} J+\lambda^{2} I\right)$, denoted here by $\kappa$, which is given by

$$
\kappa=\frac{\sigma_{1}^{2}+\lambda^{2}}{\sigma_{n}^{2}+\lambda^{2}}
$$

where 0 is used for $\sigma_{n}$ if $m<n$. A maximum condition number of $\kappa_{\max }$ can be guaranteed by using the damping factor given by

$$
\lambda\left(\kappa_{\max }\right)=\sqrt{\frac{\sigma_{1}^{2}-\kappa_{\max } \sigma_{n}^{2}}{\kappa_{\max }-1}} .
$$

While concern for the conditioning of the matrix $J^{T} J+\lambda^{2} I$ is a valid numeri- 
cal point, especially since the condition number of the Jacobian is squared to form the product $J^{T} J$, in practice, bounds on the joint angle velocity norm are usually a more stringent constraint so that a reasonable joint angle velocity will tend to automatically satisfy the numerical constraint of conditioning.

\section{IMPLEMENTATION OF DAMPED LEAST-SQUARES SOLUTIONS WITH MINIMUM SINGULAR VALUE ESTIMATES}

The damped least-squares solution of (1) is given by (8). While this solution can be obtained by solving (4) it is preferable to compute this solution by using the mathematically equivalent form

$$
\dot{\boldsymbol{\theta}}^{(\lambda)}=J^{T}\left(J J^{T}+\lambda^{2} I\right)^{-1} \dot{\mathbf{x}}
$$

especially when dealing with redundant manipulators. For redundant manipulators $m<n$ so that solutions based on the $m$ by $m$ matrix $J J^{T}+\lambda^{2} I$ require fewer calculations than the $n$ by $n$ matrix $J^{T} J+\lambda^{2} I$. The solution of (19) is best performed in two part solving

$$
\left(J J^{T}+\lambda^{2} I\right) \mathbf{z}=\dot{\mathbf{x}}
$$

for $\mathbf{z}$ and then substituting it into the Equation ${ }^{20}$

$$
\dot{\boldsymbol{\theta}}^{(\lambda)}=J^{T} \mathbf{z}
$$

In this manner an explicit inversion can be avoided by using an appropriate factorization; since $J J^{T}+\lambda^{2} I$ is symmetric, the Cholesky decomposition is an ideal choice.

The solution of (20) presents an ideal place to obtain an estimate of the minimum singular value of $J$. Since the Cholesky decomposition of $J J^{T}+\lambda^{2} I$ is already available, the solution for an additional right-hand side chosen for minimum singular value estimation represents a minimal amount of additional computation. For this reason the solution of $(20)$ is modified to the partitioned matrix equation

$$
\left(J J^{T}+\lambda^{2} I\right)\left[\mathbf{z}: \hat{\mathbf{u}}_{\mathbf{m}}^{\prime}\right]=\left[\dot{\mathrm{x}}: \hat{\mathbf{u}}_{\mathbf{m}}\right]
$$

where $\hat{\mathbf{u}}_{m}$ is a unit vector designed to optimize the estimate of the minimum singular value. The vector $\hat{\mathbf{u}}_{\mathbf{m}}$ can be written in terms of the basis specified by the output singular vectors of $J$ as

$$
\hat{\mathbf{u}}_{\mathbf{n}}=\sum_{i=1}^{m} a_{i} \mathbf{u}_{i}
$$

were the $a_{i}$ denote the component along the respective singular vector. The solution for $\hat{\mathbf{u}}_{\mathbf{w}}^{\prime}$ can then be obtained from (22) as 


$$
\hat{\mathbf{u}}_{\mathbf{m}}^{\prime}=\left(J J^{T}+\lambda^{2} I\right)^{-1} \hat{\mathbf{u}}_{\mathbf{m}}=\sum_{i=1}^{m} \frac{a_{i}}{\sigma_{i}^{2}+\lambda^{2}} \mathbf{u}_{\mathbf{i}}
$$

If $\hat{\mathbf{u}}_{\mathbf{m}}$ has a strong component in the direction of $\mathbf{u}_{\mathbf{m}}$ so that $a_{m} \approx 1$, then

$$
\left\|\hat{\mathbf{u}}_{=}^{\prime}\right\| \approx \frac{1}{\sigma_{m}^{2}+\lambda^{2}}
$$

and for a known value of $\lambda$ an estimate of the minimum singular value can be obtained. This technique is an application of the singular value estimation procedure used in numerical analysis packages involving matrix computations $^{21}$ and is based on the inverse iteration method for computing eigenvalues. A proof of its ability to provide an estimate of the minimum singular value to any specified tolerance is given in Ref. 22 .

In order to obtain a good estimate of the minimum singular value, the vector $\hat{\mathbf{u}}_{\mathbf{m}}$ must contain a significant component within the subspace spanned by the singular vectors associated with small singular values. In particular, for those cases where there is only one minimum singular value, $\hat{u}_{\mathbf{m}}$ must be nearly identical (within a sign) to $\mathbf{u}_{\mathbf{m}}$. This vector is maintained by first setting $\hat{\mathbf{u}}_{\mathbf{m}}$ exactly to $u_{m}$ before starting the trajectory. Then at every computation cycle time when (22) is solved the vector $\hat{u}_{\mathbf{m}}$ is replaced by a normalized version of $\hat{\mathbf{u}}_{\mathrm{z}}^{\prime}$. By comparing (23) with (24) it is easy to show that $\hat{\mathbf{u}}_{-}^{\prime}$ will always have an even larger relative component along the minimum singular vector than the original estimate $\hat{\mathbf{u}}_{\mathbf{m}}$. In particular, for $\hat{\boldsymbol{u}}_{\mathbf{m}}$ the relative component of $\boldsymbol{u}_{\mathbf{m}}$ to any other singular vector $u_{i}$ is given by the ratio $a_{m} / a_{i}$. For $\hat{\mathbf{u}}_{-}^{\prime}$ this ratio is given by

$$
\frac{a_{m}^{\prime}}{a_{i}^{\prime}}=\left(\frac{a_{m}}{a_{i}}\right)\left(\frac{\sigma_{i}^{2}+\lambda^{2}}{\sigma_{m}^{2}+\lambda^{2}}\right)
$$

Since $\sigma_{i}$ is always greater than or equal to $\sigma_{m}$ by definition, the second factor in (26) is always greater than or equal to one thus improving the estimate of the minimum singular vector. In this manner, as the singular vector associated with the minimum singular values rotates, $\hat{\mathbf{u}}_{\mathbf{m}}$ is able to rotate along with it, always maintaining a strong component along that direction. The perturbation bounds on the rotation of singular vectors discussed in Ref. 23 guarantee that the subspace associated with the small singular values is well-behaved.

The estimate for the minimum singular value for $J$ can now be used to set an appropriate damping factor using the equations of the previous section. There is an apparent circular dilemma in that $\lambda$ must be known in order to estimate $\sigma_{m}$ from (25) yet the reason for calculating $\sigma_{m}$ is to be able to set $\lambda$ to an appropriate value. This dilemma is resolved by setting the damping factor using the previous estimate of the minimum singular value. Since singular values are well conditioned, the bounds on their rates of change, discussed in Ref. 23, guarantee that the change in the minimum singular value will be small during the computation time interval so that the above value of the damping factor can be used. 


\section{NUMERICAL FILTERING OF SINGULAR COMPONENTS}

One of the limitations of the damped least-squares approach is a result of the uniform damping factor applied to all singular values. From a physical point of view it would be desirable if only those components of the end effector velocity which are difficult to achieve would be damped when a manipulator is in a singular configuration. The effect of such a formulation would be similar to a continuous version of the truncated SVD solution where error introduced by the damping of acceptable singular values is eliminated. The advantages of such a formulation are the removal of unnecessary end effector tracking errors due to the presence of small singular values even though the desired end effector velocity has no component in the singular directions. Such a solution requires replacing the identity matrix in (22) with a more general matrix designed to selectively filter different components to varying degrees. This approach is analogous to the numerical filtering used in the solution of Fredholm equations for problems involving indirect sensing ${ }^{24}$ and electromagnetics. ${ }^{25}$

The estimate $\hat{u}_{\mathbf{n}}$ can be used in order to achieve a solution in which the components associated with the small singular values are damped more than the others. By replacing (22) with

$$
\left(J J^{T}+\alpha^{2} \hat{u}_{m} \hat{u}_{=}^{T}+\lambda^{2} I\right)\left[z \vdots \hat{u}_{m}^{\prime}\right]=\left[\dot{x} \vdots \hat{u}_{m}\right]
$$

the filter gain $\alpha$ can be used to provide further damping of the singular components in addition to the overall damping factor $\lambda$. By using (27) in place of $(22)$ the solution norm is now given by

$$
\left\|\dot{\theta}^{(\alpha, \lambda)}\right\|^{2} \approx \sum_{i=1}^{m-1} \dot{x}_{i}^{2}\left[\frac{\sigma_{i}}{\sigma_{i}^{2}+\lambda^{2}}\right]^{2}+\dot{x}_{m}^{2}\left[\frac{\sigma_{m}}{\sigma_{m}^{2}+\alpha^{2}+\lambda^{2}}\right]^{2}
$$

where the accuracy of the approximation depends on the accuracy to which $\hat{u}_{\text {m }}$ matches $u_{m}$. As a manipulator approaches a singularity, it is the last term in (28) which induces the high joint velocities. Without the filter gain $\alpha$, the overall damping factor $\lambda$ would be forced to increase, unnecessarily damping the well-behaved components in the summation term and resulting in an unnecessary end effector tracking error. By introducing an extra degree of freedom as to how damping is applied, the filter gain $\alpha$ can be set based on the estimate of the minimum singular value obtained from (25) which then allows a reduction in the overall damping factor. Note that these advantages are present regardless of the value of $\dot{x}_{m}$.

The computation of an appropriate overall damping factor $\lambda$ is based on establishing an effective singular value for the specific commanded end effector velocity. This effective singular value is based on modeling the commanded end effector velocity $i$ as being composed of two components, one within the possibly singular subspace defined by $\hat{u}_{-}$and denoted by $\dot{\boldsymbol{x}}_{\mathbf{m}}$, and the other component in the remaining orthogonal subspace denoted by $\dot{\boldsymbol{x}}_{0}$. 
These two components are easily calculated using

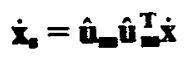

and

$$
\dot{\mathbf{x}}_{0}=\dot{\mathbf{x}}-\dot{\mathbf{z}} .
$$

The additional information required for establishing an effective singular value is obtained from the vector $z$ calculated from the previous computation interval. From (27) one can write $z$ as

$$
z \approx\left[\sum_{i=1}^{m-1} \frac{\dot{x}_{i} u_{i}}{\sigma_{i}^{2}+\lambda^{2}}\right]+\frac{\dot{z}_{i}}{\sigma_{m}^{2}+\alpha^{2}+\lambda^{2}}
$$

where once again the accuracy of the approximation depends on the accuracy to which $\hat{u}_{\text {. }}$ matches $u_{\text {. }}$. All of the values in the last term of (31) are known so that the portion of $\mathbf{z}$ which results from $\dot{\Sigma}_{0}$, denoted here by $z_{0}$, can be calculated from

$$
\mathbf{z}_{\mathbf{0}}=\mathbf{z}-\frac{\dot{\mathbf{z}}_{\mathbf{m}}}{\sigma_{m}^{2}+\alpha^{2}+\lambda^{2}}
$$

A measure of the effective singular value outside of the singular subspace, denoted by $\sigma_{o}$, can then be obtained by modeling the summation term in (31) by a one-dimensional space resulting in

$$
\left\|\mathbf{z}_{0}\right\|=\frac{\left\|\dot{\mathbf{x}}_{0}\right\|}{\sigma_{0}^{2}+\lambda^{2}}
$$

or

$$
\sigma_{0}^{2}=\frac{\left\|\dot{x}_{0}\right\|}{\left\|z_{0}\right\|}-\lambda^{2}
$$

A measure of the overall effective singular value for the current commanded end effector velocity can then be obtained by comparing the components $\dot{x}_{\text {, }}$ and $\dot{z}_{0}$. In particular, the overall effective singular value, denoted by $\sigma_{e f}$ is given by

$$
\left(\frac{\|\dot{\mathbf{x}}\|}{\sigma_{e f f}}\right)^{2}=\left(\frac{\left\|\dot{x}_{1}\right\|}{\sigma_{m}}\right)^{2}+\left(\frac{\left\|\dot{x}_{0}\right\|}{\sigma_{o}}\right)^{2} .
$$

The entire motivation for developing (35) is to provide a means of incorporating information about how the current commanded end effector velocity will 
affect the resulting solution. In effect what (35) does is provide an estimate of what the resulting joint angle velocity norm would be if no damping were applied. This effective singular value is used in place of the minimum singular value in the equations of Section III for calculating an appropriate overall damping factor.

The approach outlined in this section allows the manipulator to differentiate between situations where an arbitrary or only a particular end effector velocity is difficult to achieve. The former case is only a function of the manipulator's configuration and can be identified by examining the minimum singular value of the Jacobian. While this case is simpler to determine, algorithms based on this limited information can result in unnecessary degradation of performance in the latter case where the commanded end effector velocity has no component in the singular subspace. With the numerical filtering approach the damping factor $\lambda$ will be zero in this case so that no end effector tracking error will be introduced. Note that the implicit inversion of (27) is still well conditioned since a non-zero value of $\alpha$ damps the ill-conditioned singular subspace components and does not affect the end effector tracking. While the numerical filtering approach does require some additional calculations, they do not appreciably increase the overall computation time. This is due to the fact that solution of the matrix equations $(20)$ and $(21)$ require on the order of $O\left(n^{3}\right)$ floating point operations whereas calculation of $\sigma_{\text {eff }}$ from (35) requires only a few vector operations which can be performed in linear time.

\section{SIMULATION RESULTS}

This section presents computer simulation results using the formulations presented above. The techniques discussed are fully general and have been implemented for use with any arbitrary three-dimensional manipulator. Simulation results are initially presented for a planar two-dimensional manipulator in order to facilitate the visualization of the associated singular vectors with the results for the three-dimensional simulations presented in the following section. In all cases, resolved motion rate control, as defined by (1), is used as a representative example of the characteristics for these types of solutions. Simulations using resolved acceleration control have produced similar results. ${ }^{23}$ The commanded velocity for the end effector, $\dot{\boldsymbol{x}}_{c}$, is composed of the desired velocity plus a position error term given by

$$
\dot{\mathbf{x}}_{\mathbf{c}}=\dot{\mathbf{x}}_{\mathrm{d}}+k_{\mathrm{p}}\left(\mathbf{x}_{\mathrm{d}}-\mathbf{x}_{\mathrm{q}}\right)
$$

where $x_{4}$ and $x_{k}$ are the desired and actual positions of the end effector and $k_{p}$ is the position error feedback grain. The normalized end effector tracking error, $\dot{x}_{e r}$, is defined as

$$
\dot{\boldsymbol{x}}_{e r}=\frac{\left\|\dot{\mathbf{x}}_{\mathbf{c}}-\boldsymbol{J} \dot{\boldsymbol{\theta}}^{(\lambda)}\right\|}{\left\|\dot{\mathbf{x}}_{\boldsymbol{r}}\right\|}
$$




\section{Two-Dimensional Results}

The two-dimensional simulations for the control of manipulators through singular configurations were performed on the two-link planar manipulator depicted in Figure 2. The manipulator is shown in its initial configuration along with the desired trajectory for the end effector. As illustrated, the link lengths are 110 and $100 \mathrm{~cm}$ for the first and second links, respectively. The desired trajectory is $200 \mathrm{~cm}$ square, being offset by $10 \mathrm{~cm}$ from the base of the manipulator. By commanding the manipulator to travel along the square $A B C D$, the reach singularity is encountered along a significant part of the trajectory, including the entire segment from $B$ to $C$ for which the desired end effector position is kinematically unachievable except for the midpoint of segment $\mathrm{BC}$. In addition, the trajectory is designed to force the manipulator to go through the internal singularity at the midpoint between points $\mathrm{D}$ and $\mathrm{A}$. This trajectory was intentionally chosen outside of the achievable workspace in order to illustrate the smooth operation of the techniques presented here. While such trajectories are not planned in controlled environments, they do occur in cases where teleoperators are employed. The desired velocity is uniform in magnitude at one $\mathrm{cm}$ per computation interval, thus resulting in discontinuities in direction at the vertices of the square. While these discontinuities result in a physically impossible trajectory, they were included in order to emphasize the effect that the direction of the commanded end effector velocity has on the resulting solution. The position error feedback gain $k_{p}$, is set at the relatively low value of 0.1 in order to keep the norm of the

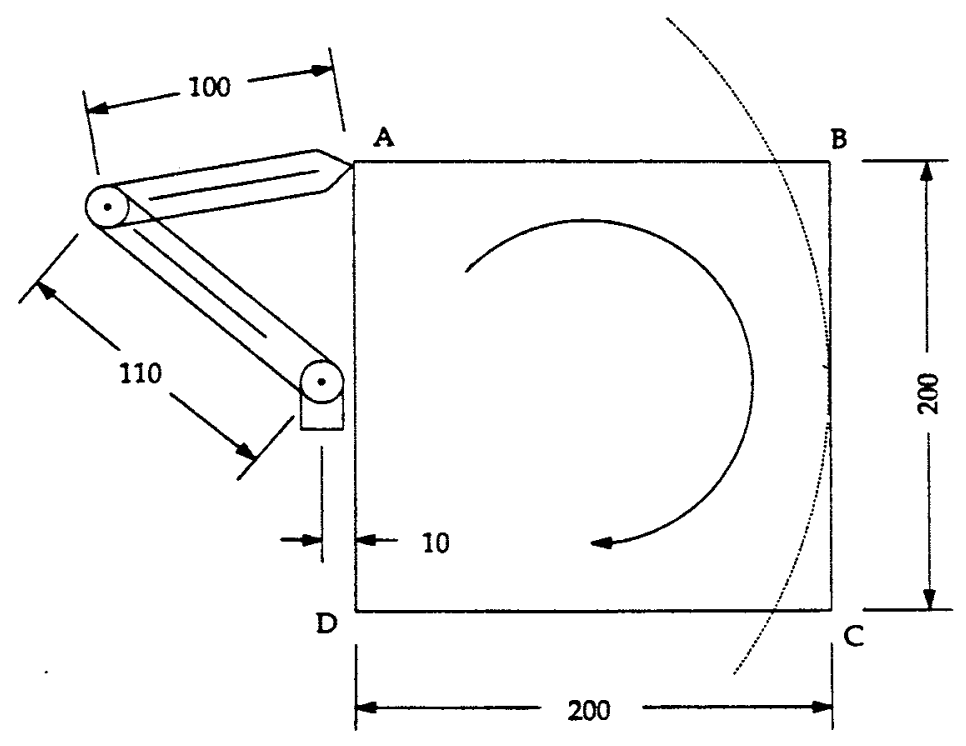

Figure 2. Two-link planar manipulator and desired trajectory used for the twodimensional simulations. The maximum reach of the manipulator is shown in dotted lines. Lengths are in centimeters. 
commanded velocity within the range of 1 to $2.2 \mathrm{~cm}$ per computation interval since a maximum position error of approximately $23 \mathrm{~cm}$ will occur when the manipulator is commanded to be at points $B$ or $C$.

The simulation results for the three cases of a constant damping factor, a variable damping factor, and the numerical filtering formulation are presented in Figure 3. A maximum damping factor of 25 was calculated from (10) in order to maintain the actual joint velocity norm to within 0.05 radians per computation interval. For the variable damping factor simulation $\lambda$ is computed from (14) based on a desired normalized solution norm, $\dot{\theta}_{\max }$, of 0.02 which also results in a maximum damping factor of 25 and an actual solution norm bound of 0.05 radians per computation interval. In order to provide a valid comparison the numerical filtering simulation also uses a maximum overall damping factor, $\lambda$, of 25 which is computed from (14) using $\sigma_{\text {eff }}$, however, in place of $\sigma_{\min }$. The filter gain, $\alpha$, is computed from (14) using the estimate of the minimum singular value.

The results for all three cases are superior to any formulation relying on traditional pseudoinverse type solutions since the transition from a full rank to a singular configuration for pseudoinverse type solutions results in unacceptably high joint velocities, oscillations, and poor end effector tracking. The damped least-squares solution, however, results in smooth transitions and velocity bounds which can be set $a$ priori. The normalized end effector tracking error is much as would be expected. The largest errors occur at points $B$ and $C$ along the trajectory. The dip in the error at the midpoint between $B$ and $C$ is due to the fact the commanded end effector velocity is directed increasingly along the singular vector associated with the non-zero singular value. The notch in the end effector velocity error occurring just after point $C$ marks the position at which the commanded end effector position matches the reach of the manipulator and it can now begin to come out of the singularity. The overshoot in the end effector tracking as the manipulator comes out of its reach singularity is due to the fact that the commanded end effector velocity has a strong component in the direction of the now small singular value. It is at this point that the peak joint velocity is reached in getting the manipulator out of the singular configuration.

The constant damping factor case has the undesirable characteristic of end effector tracking errors along the entire trajectory, even when the manipulator is far from a singular configuration. In addition, a very large error occurs near the internal singularity midway between points $D$ and $A$ even though the manipulator is only singular at a single point and at that point there is no component of desired end effector velocity in the direction of the singular vector associated with the zero singular value. The use of a variable damping factor based on an estimate of the minimum singular value is designed to alleviate some of the difficulties associated with the constant damping factor solution, namely, that of end effector errors in well-conditioned configurations. The variable damping factor simulation results illustrate that, as expected, the end effector velocity error can be reduced to near zero outside of the proximity of singular configurations. These results are particularly evident in 

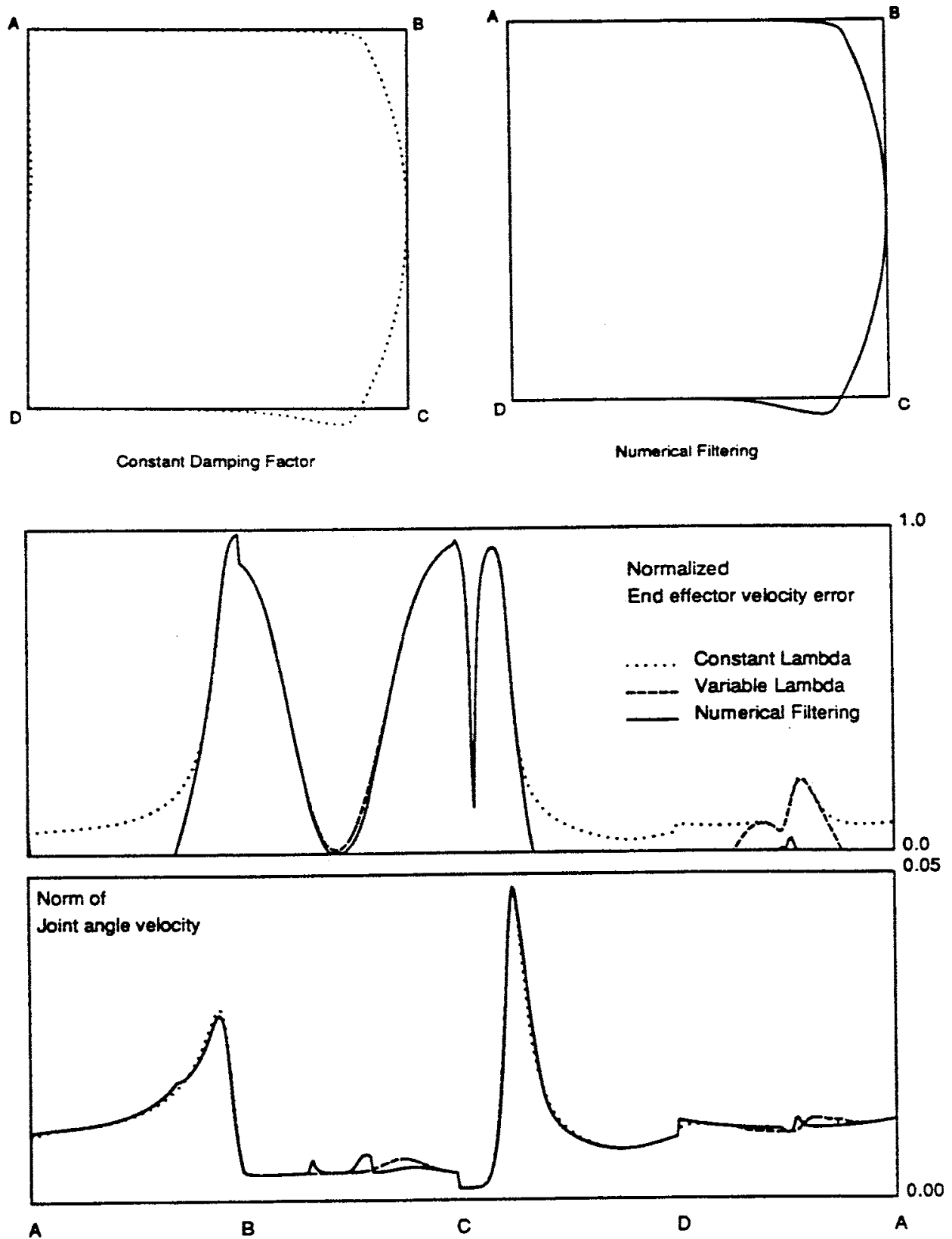

Figure 3. Results of the two-dimensional simulation showing the end effector trajectory along with plots of the end effector velocity error and joint velocity. 
the neighborhood of points $A$ and $D$ where the error is reduced to zero without an appreciable change in the joint velocity. By using this formulation the desirable properties of predictable performance at singularities can be coupled with improved performance away from singularities. Note, however, that there is still a significant amount of end effector tracking error in the neighborhood of the internal singularity. This error is due to the fact that all components of the desired end effector velocity are equally damped. Thus the non-singular component is damped by the maximum damping factor since the minimum singular value is zero. This degre of damping is clearly unnecessary as can be seen from the still relatively low joint velocity norm.

By applying the numerical filtering technique the manipulator can treat the various components of the commanded end effector velocity with different damping factors, thus further improving the performance at singular configurations. The most dramatic difference in performance with numerical filtering as opposed to treating all components equally occurs in the neighborhood of singularities. In particular, at the internal singularity midway between points $D$ and $A$, the end effector error is significantly reduced from a peak value of $22 \%$ of the commanded velocity to less than $4 \%$ and from an integral error of $9.94 \mathrm{~cm}$ to 0.25 as compared to the variable damping factor case. Note that this improvement occurs without violating any of the constraints on the joint angle velocities. The reason for the improvement lies in the fact that the numerically filtered solution more accurately reflects the physical situation of the manipulator. Proximity to a singularity only reflects loss of motion in those directions associated with the corresponding singular vectors. Treating all directions equally only induces unnecessary errors in the nonsingular directions. The portion of this trajectory through the internal singularity is meant to emphasize the importance of this fact, namely that manipulators can physically pass through singular configurations without inducing high joint velocities or end effector tracking errors. Utilizing such information will result in larger effective workspaces and improved performance.

\section{Three-Dimensional Results}

The three-dimensional simulation was performed for the familiar PUMA robot geometry as a representative example, the Denavit and Hartenberg parameters for which are presented in Table I. The simulations were conducted through three different types of singularities which are illustrated in Figure 4. The singular configurations of the PUMA robot are well-known and can be identified by simple geometric properties. The first type, which is perhaps most commonly encountered, is associated with the loss of a rotational velocity component. Known as the wrist singularity, it occurs when joint angle five is equal to zero thus aligning the other two axes of the wrist. This singularity is present throughout the workspace of the manipulator since it is not associated with any of the three primary positioning joint angle variables. The second type of singularity is known as a shoulder singularity and occurs when the wrist 
Table 1. The Denavit and Hartenberg parameters for the PUMA robot used in the three-dimensional simulations.

\begin{tabular}{cccc}
\hline link & length (m) & offset $(\mathrm{m})$ & twist (deg) \\
\hline 1 & 0.000 & 0.000 & -90 \\
2 & 0.432 & 0.149 & 0 \\
3 & -0.020 & 0.000 & 90 \\
4 & 0.000 & 0.433 & -90 \\
5 & 0.000 & 0.000 & 90 \\
6 & 0.000 & 0.056 & 0 \\
\hline
\end{tabular}

of the manipulator is located along the axis of the shoulder. In this configuration, linear velocities toward or away from the base are unachievable. The third type of singularity, which also results in the loss of a linear velocity component, occurs when the elbow joint angle is equal to zero thus aligning links two and three. This singularity, known as the elbow singularity, is analogous to the reach singularity of a two-dimensional manipulator. Note that the particular version of the elbow singularity illustrated in Figure 4 is unique in that the shoulder and wrist singularities are also present, thus resulting in a triple singularity.

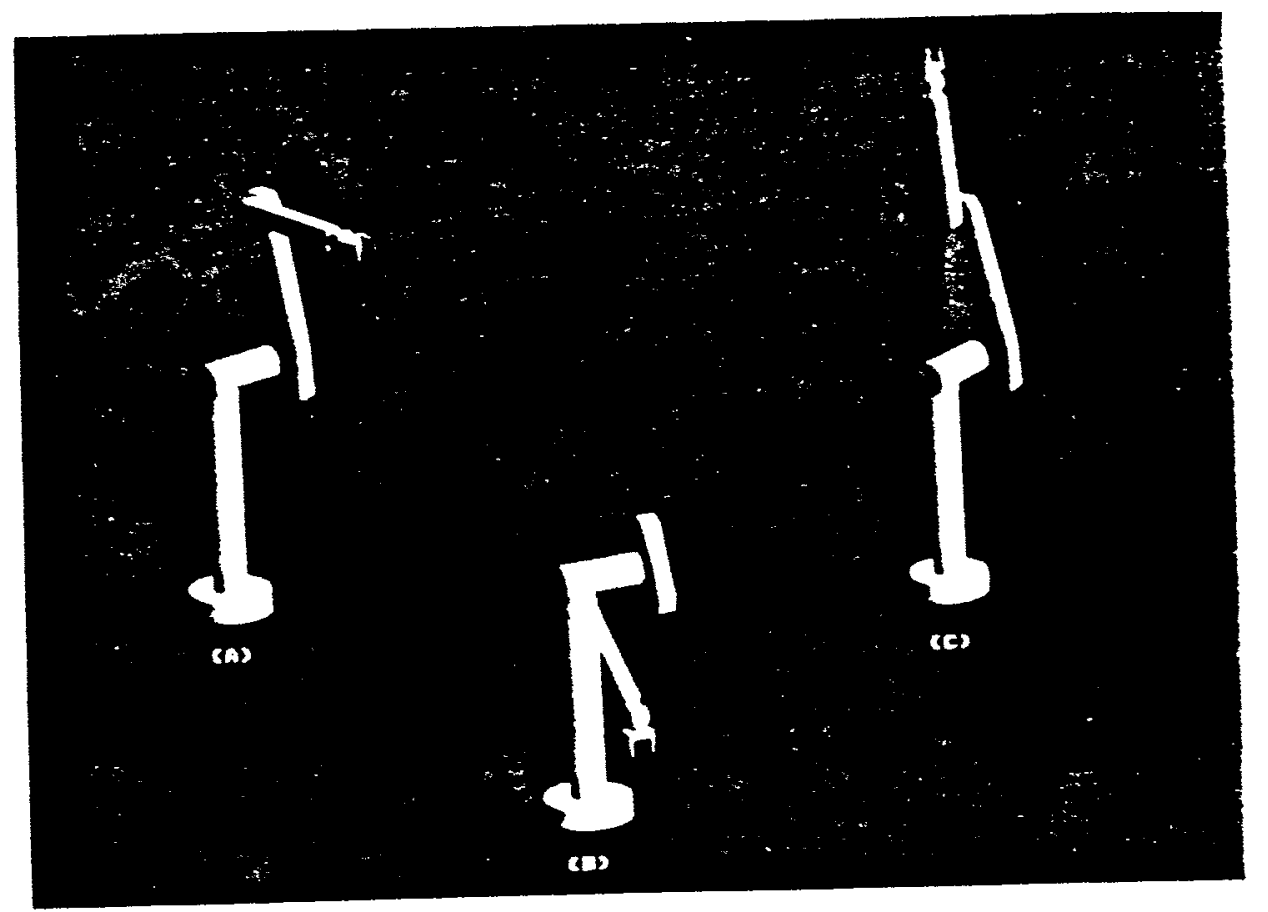

Figure 4. Three different singular configurations for the PUMA robot: (a) wrist singularity; (b) shoulder singularity; (c) triple singularity. 


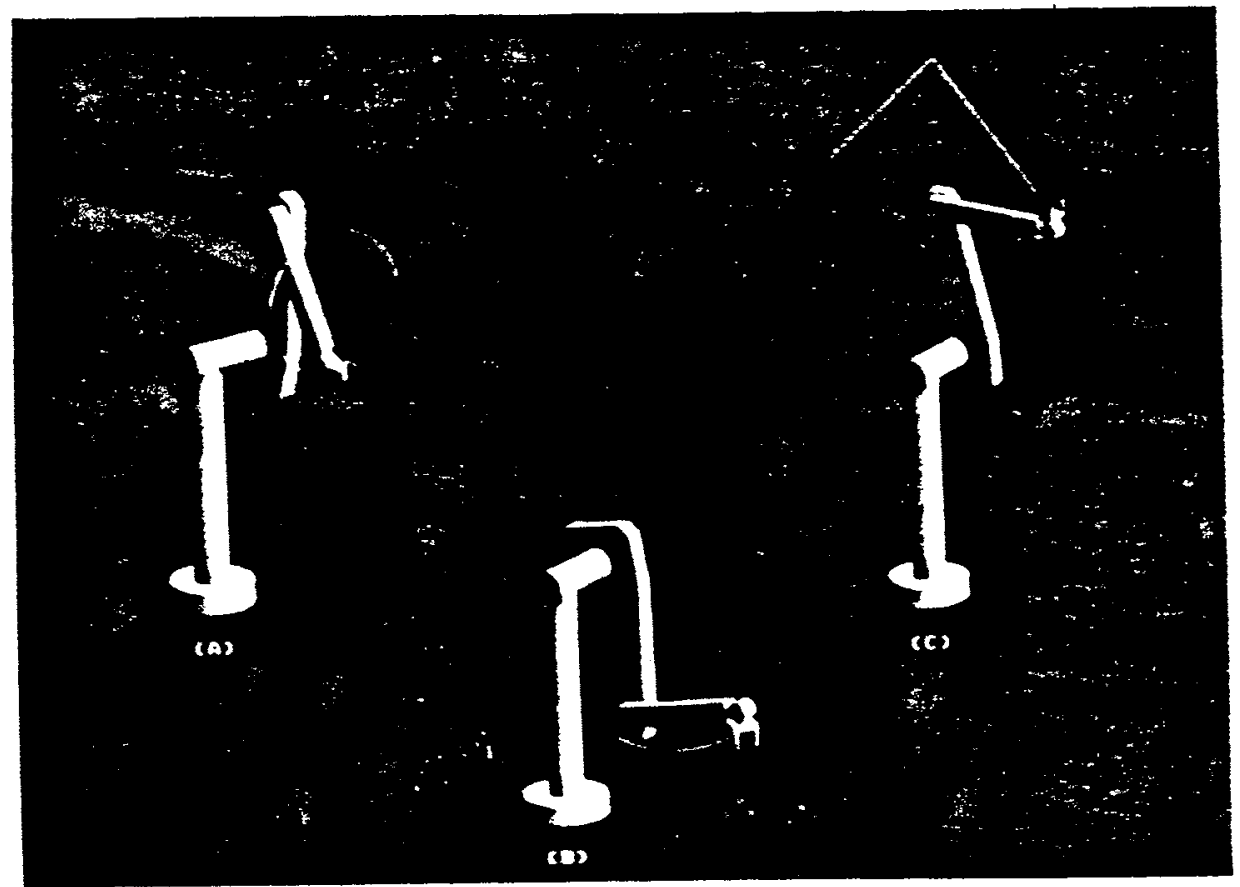

Figure 5. Three trajectories and starting configurations which pass through the singular configurations of Figure 4.

The three trajectories used to pass through these singular configurations are presented in Figure 5 along with the starting configurations. All three trajectories have the commanded singular configuration as the midpoint of the trajectory. Trajectory $A$ is a circle with a constant end effector velocity magnitude of $1.57 \mathrm{~mm}$ per computation interval. Trajectory B is composed of cosine terms in the positional variables with a maximum end effector velocity norm of $1.57 \mathrm{~mm}$ per computation interval. Trajectory $C$ is composed of two linear segments with a constant end effector velocity norm of $\sqrt{2} \mathrm{~mm}$ per computation interval. The commanded end effector velocity is given by (36) with a position error feedback gain, $k_{p}$, of 0.5 .

The results for the above three trajectories using the constant damping factor formulation and the numerical filtering approach are presented in Figures 6 to 8 . The performance of the variable damping factor technique is bracketed by these two cases and is therefore omitted. All simulations were conducted with a maximum damping factor, $\lambda$, of 0.08 with an $\alpha$ of 0.01 for the numerical filtering formulation. The graphed results of the end effector error and joint velocity over the trajectory are accompanied by selected configurations of the manipulator along the trajectory. These configurations are equally spaced in time, one every 25 computation cycle intervals, and depict the manipulator as line segments connecting the origins of the various link coordinate systems in a parallel projection. 

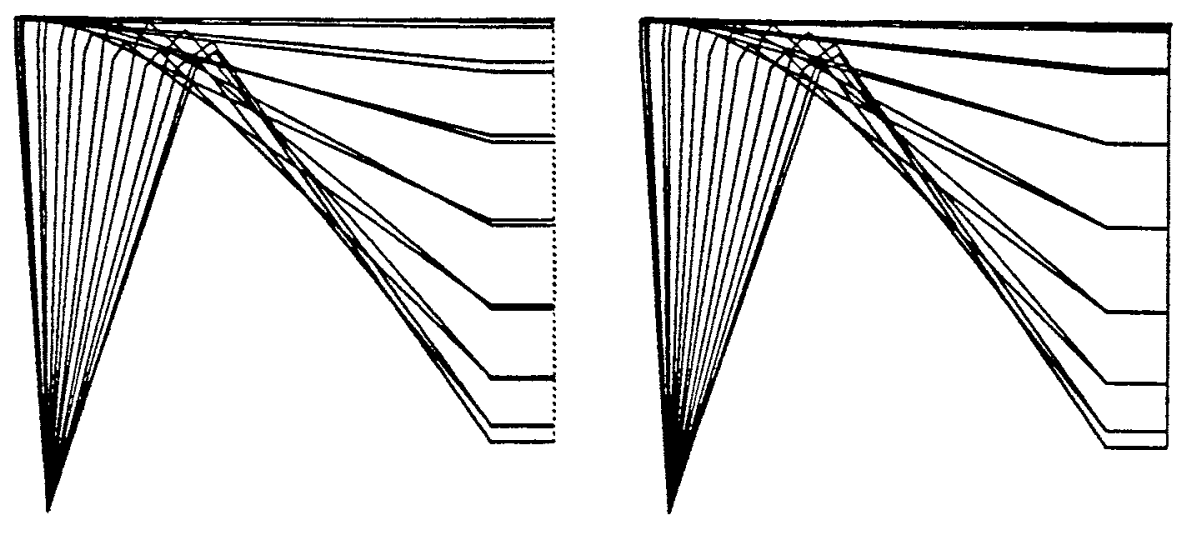

condent Oenoung Factor
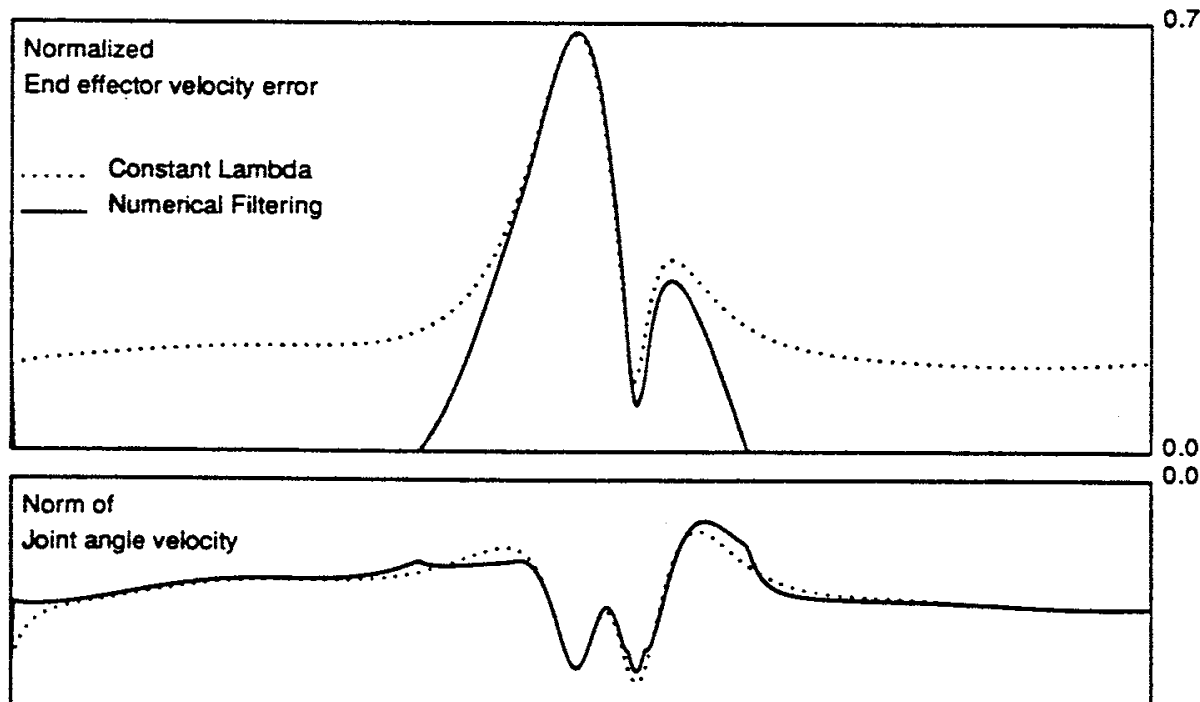

0.00

Trajectory A

Figure 6. Results of PUMA robot simulation for trajectory A showing selected manipulator configurations and the end effector trajectory along with plots of the end effector velocity error and joint velocity. 

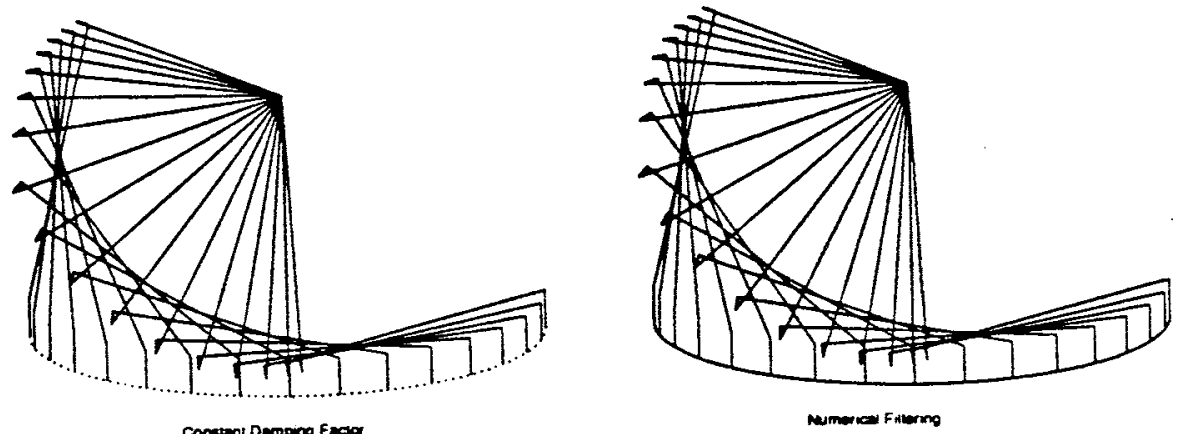

Conetent Damping factor

0.6

Normalized

End effector velocity error

Constant Lambda

Numerical Filtering
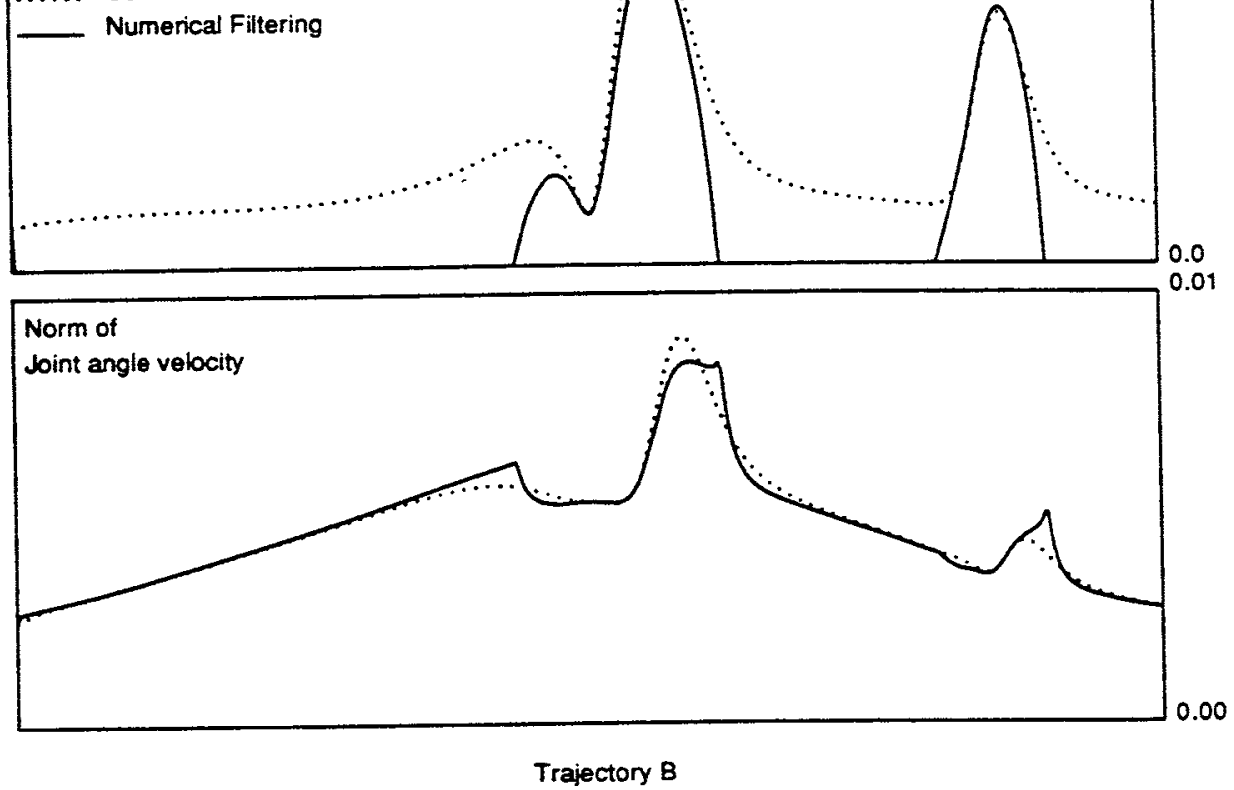

Figure 7. Results of PUMA robot simulation for trajectory B showing selected manipulator configurations and the end effector trajectory along with plots of the end effector velocity error and joint velocity.

The results for trajectory $A$ are presented in Figure 6 and illustrate the typical behavior of these types of formulations. The singularity is clearly encountered at the top of the circular trajectory since the hand of the manipulator is aligned with the forearm. The constant damping factor formulation has a nominal end effector velocity error of $15 \%$ of the commanded velocity which is present throughout the trajectory and increases in the 

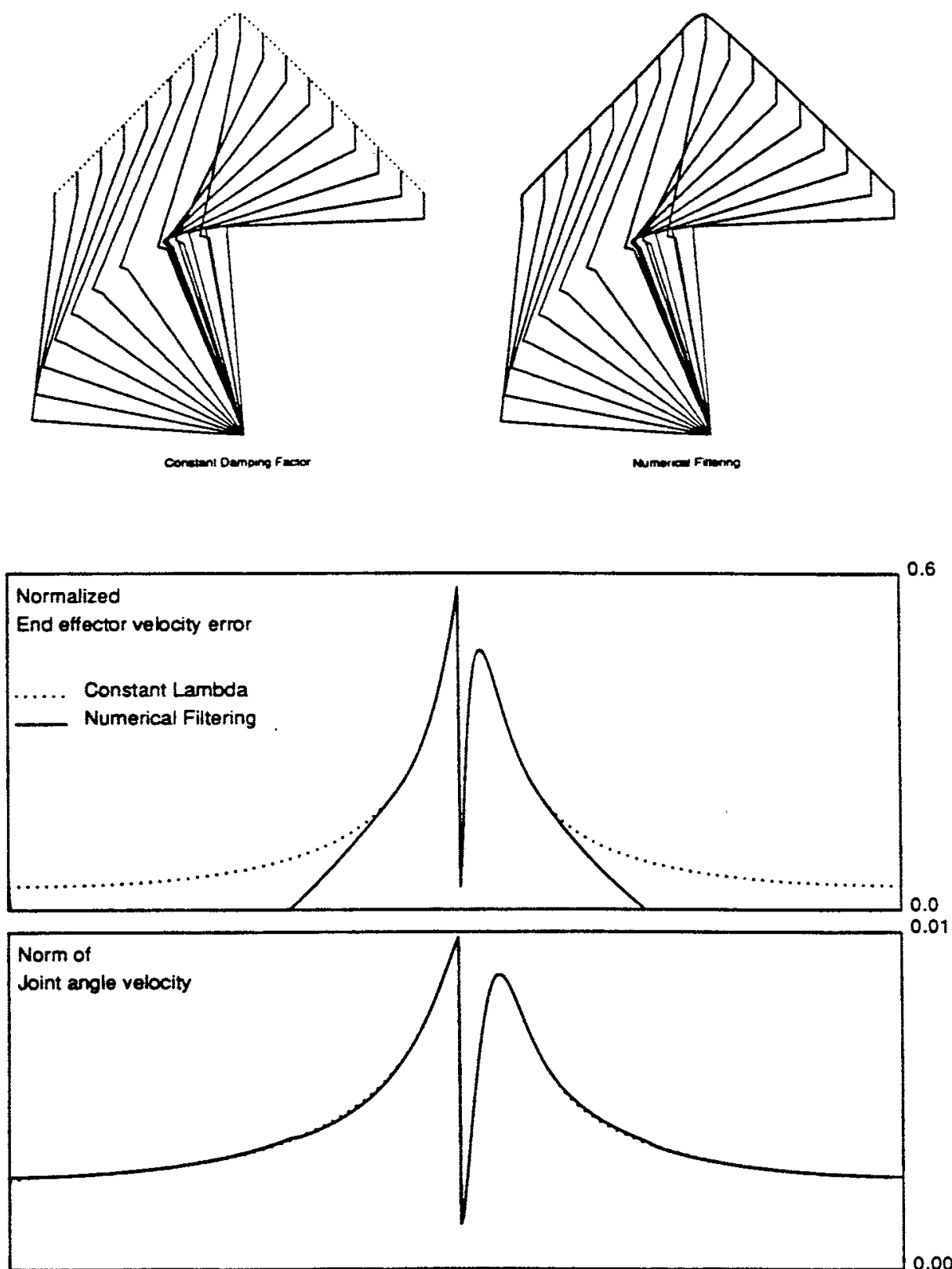

Trajectory C

Figure 8. Results of PUMA robot simulation for trajectory $C$ showing selected manipulator configurations and the end effector trajectory along with plots of the end effector velocity error and joint velocity. 
proximity of the singularity. The notch in the error is once again associated with a larger component along the nonsingular directions. The position error is also clearly evident from the configuration plot of the manipulator since the projections of the end effector for the upward and downward motion of the manipulator do not coincide. The results for the numerical filtering approach show that like the two-dimensional simulation, the end effector error outside of the singular region has been reduced to near zero. The configuration plot collaborates this data by having coincident end effector positions except near the top of the trajectory. The only remaining end effector error is due to the lost degree of rotational velocity at the wrist.

The results for trajectory B are presented in Figure 7. Once again a nominal end effector velocity error is present throughout the trajectory for the constant damping factor solution with a notched peak at the shoulder singularity. The single peak near the end of the trajectory is due to the proximity of a wrist singularity which is clearly evident in the manipulator configuration plot where once again the hand and forearm are nearly collinear. The results of the numerical filtering approach show that only the physically unachievable velocities associated with the singular vectors of small singular values account for the end effector errors which are present. The interesting feature to note about this trajectory is that the minimum singular value estimation technique must switch very rapidly from following the singular vector associated with the shoulder singularity to that associated with the wrist singularity.

The final trajectory, $\mathrm{C}$, represents the worst-case situation for this manipulator geometry where a triple singularity is encountered. These results are presented in Figure 8 . The notched peak at the singularity is a bit more pronounced due to the higher order of the singularity. The broadening of the area around the singular configuration for which there is a non-zero end effector velocity error for the numerical filtering case is also a result of the higher order singularity. This behavior is due to the fact that a higher dimensional space of end effector velocities are physically more difficult to achieve. Thus the solution still accurately reflects the state and capabilities of the manipulator under the imposed joint velocity constraints.

It is instructive to examine the behavior of the singular values for trajectory $\mathrm{C}$ in order to gain insight into the behavior of the technique for maintaining an estimate of the minimum singular value under these worst case conditions. A plot of all six singular values for the manipulator's Jacobian for the entire trajectory is presented in Figure 9. The notch in the three smallest singular values at the center of the trajectory is where the manipulator is closest to the triple singularity. Since the manipulator is only commanded to go through the singularity at one point, it never actually reaches the completely singular configuration which can be plainly seen from the configuration plots of Figure 8. Examination of the singular vectors associated with the singular values reveals that the smallest singular value is primarily associated with the wrist singularity, the next smallest with the elbow singularity, and the third smallest primarily with the shoulder singularity. It is interesting to note that the fourth largest singular value is still relatively small compared to the two largest ones. 
Singular Values

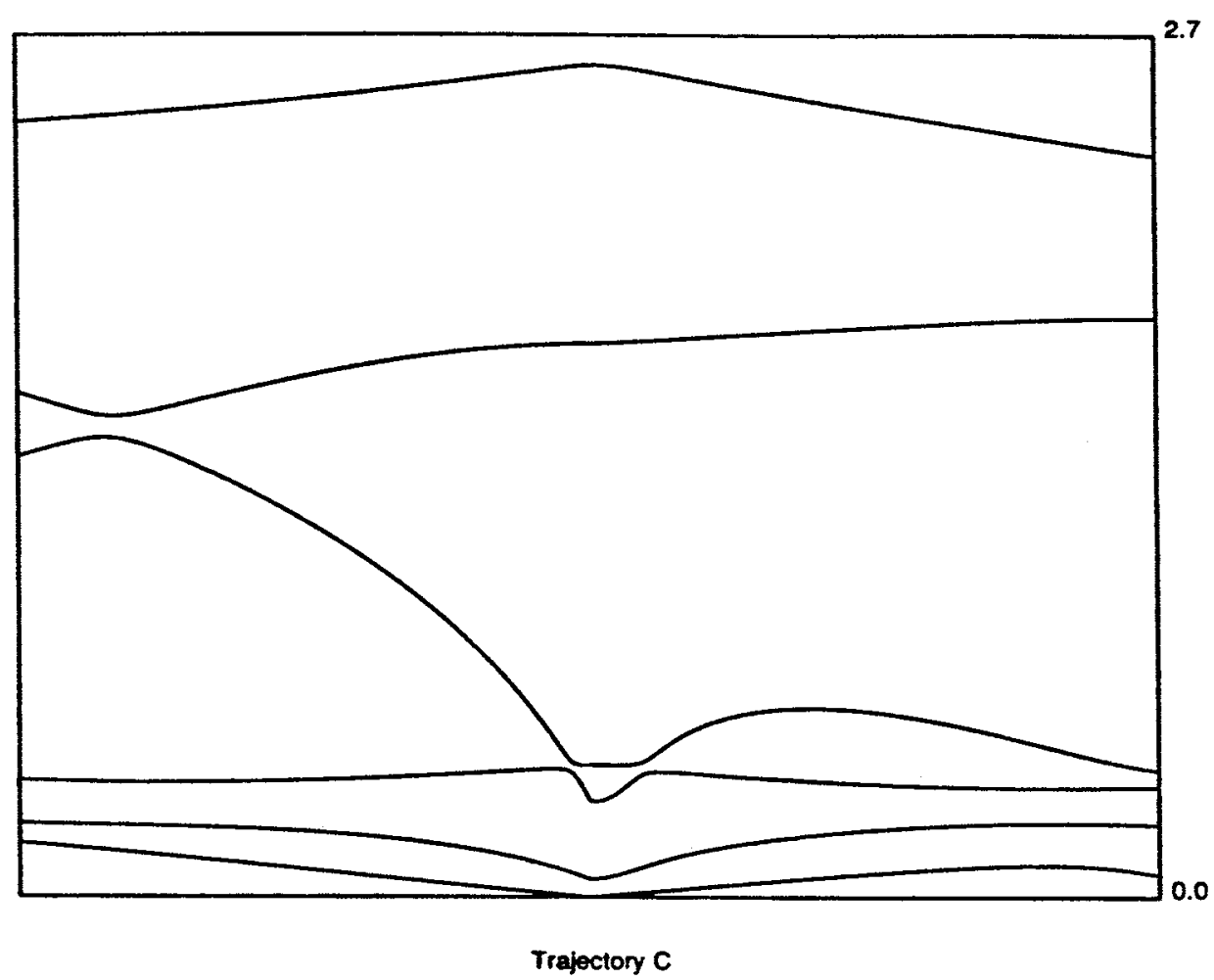

Figure 9. Singular values of the Jacobian matrix for each configuration along trajectory $\mathrm{C}$.

This is due to the fact that the linear and rotational velocity components of joints two, three, and five are coupled.

The technique for maintaining an estimate of the minimum singular value is based on the rotation bounds for singular vectors from which it is known that the most rapid rotation occurs when there are multiple minimum singular values. This case already occurred during trajectory $B$, but it occurred when the twin minimum singular values were above the threshold which would have affected the damping factor. The most critical test of the estimation technique occurs when the rapid rotation of singular vectors coincides with twin minimum singular values which are sufficiently small to have a noticeable effect on the damping factor. In order to force this case to occur, the second half of trajectory $C$ was modified to a constant position command at the reach of the manipulator with a rotational velocity command in the direction of the axis of joint 5. This modification forces the singular value associated with the elbow singularity to go to zero while increasing the singular value associated with the wrist singularity. This causes the two small singular values to cross and thus 


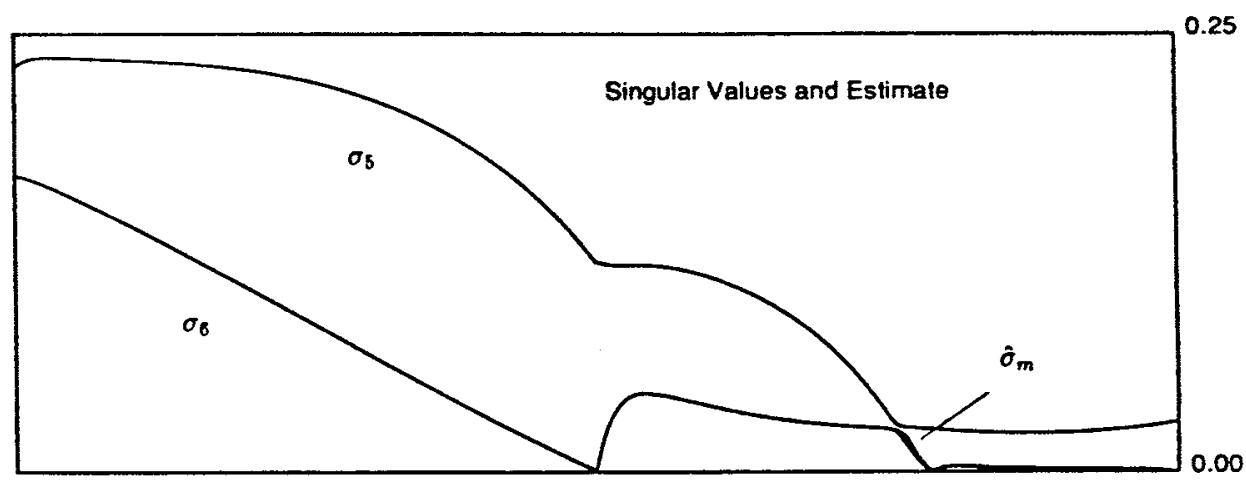

Dot Product of Estimate

with Minimum Singular Vector

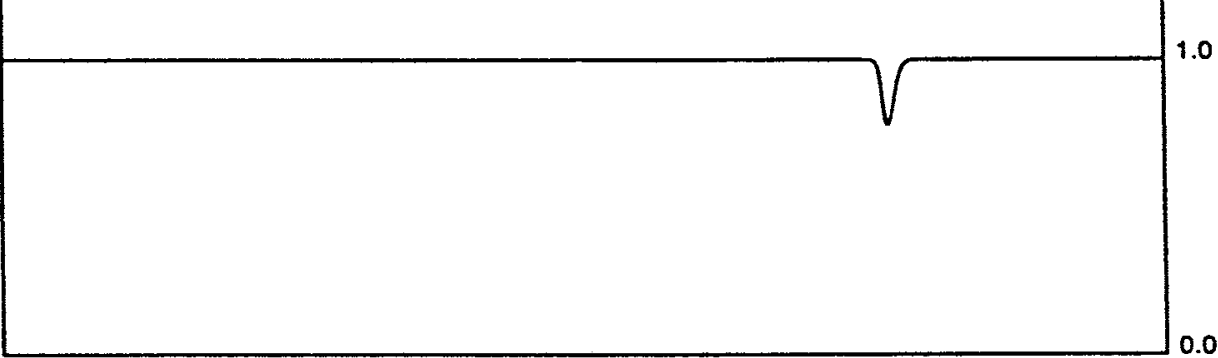

Modilied Trajectory C

Figure 10. Smallest singular values $\sigma_{5}$ and $\sigma_{6}$ and the estimate to the minimum singular value, $\hat{\sigma}_{m}$, along the modified trajectory $C$. Also shown is the dot product of the singular vectors associated with $\sigma_{0}$ and $\hat{\sigma}_{m}$. Modified trajectory $C$ has been designed as a worst case situation for rapid rotation of singular vectors.

the singular vector associated with the minimum singular value rapidly rotates its maximum value of 90 degree. A plot of the two smallest singular values for this modified trajectory is presented in Figure 10. In order to measure the performance of the estimation technique, the minimum singular value estimate is also plotted along with the dot product of the estimation vector, $\hat{\mathbf{u}}_{\mathbf{m}}$, and the singular vector associated with the minimum singular value. The only noticeable error in the estimate occurs just after the center of the trajectory when the rapid rotation is in progress. At this point the estimate is an average of the two nearly equal singular values. As the singular values separate, however, the estimation vector rapidly rotates to track the new minimum singular value. Thus even in a worst case analysis, this procedure provides a robust estimate of the minimum singular value.

\section{CONCLUSIONS}

Kinematically singular configurations are an inherent property of articulated manipulators. Using numerical techniques which account for there presence 
prevents unexpected spurious motions, oscillations, and unacceptably high joint velocities while expanding the usable workspace. The performance of the techniques illustrated here varies with the amount of information included in the formulation. In general, the more information regarding the manipulator's current state and specific trajectory, the more accurately can the formulation match the manipulator's capabilities to the requirements of the assigned task. In particular, the simplest formulation using damped least-squares solutions with a predefined constant damping factor can guarantee bounded joint velocities and smooth transitions through singular configurations, however the performance at well-conditioned configurations is unnecessarily compromised. By including a minimum singular value estimation technique into the process, knowledge of when the manipulator is near a singular configuration can be used to combine the performance of traditional inverse or pseudoinverse techniques at well-conditioned configurations with the characteristics of the damped solutions at singular configurations. Including specific information regarding the commanded end effector velocity permits the filtering of singular components to be separated from well-conditioned components thus achieving the physically realizable limits of manipulator performance for the given task.

\section{References}

1. D. R. Baker and C. W. Wampler, "Some facts concerning the inverse kinematics of redundant manipulators," 1987 IEEE Intemational Conference on Robotics and Automation, Raleigh, N.C., March-April 1987, pp. 604-609.

2. B. Huang and V. Milenkovic, "On an algorithm negotiating wrist singularities," Robots 11/17th Int. Sym on Ind. Robots, Chicago, IL, April 26-30, 1987, pp. 13.1-13.6.

3. H. Asada and J. A. Cro Granito, "Kinematic and static characterization of wrist joints and their optimal design," 1985 IEEE Intemational Conference on Robotics and Automation, St. Louis, March 1985, pp. 244-250.

4. R. V. Mayorga and A. K. C. Wong, "A singularities avoidance method for the trajectory planning of redundant and nonredundant robot manipulators," 1987 IEEE International Conference on Robotics and Automation, Raleigh, N.C., March-April 1987, pp. 1707-1712.

5. E. W. Aboaf and R. P. Paul, "Living with the singularity of robot wrists," 1987 IEEE Intermational Conference on Robotics and Automation, Raleigh, N.C., March-April 1987, pp. 1713-1717.

6. R. Dubey and J. Y.S. Luh, "Redundant robot control for higher flexibility," 1987 IEEE Intemational Conference on Robotics and Automation, Raleigh, N.C., March-April 1987, pp. 1066-1072.

7. M. Sampei and K. Furuta, "Robot control in the neighborhood of singular points," 1987 IEEE Intemational Conference on Robotics and Automation, Raleigh, N.C., March-April 1987, pp. 1696-1700.

8. D. E. Whitney, "Resolved motion rate control of manipulators and human prostheses," IEEE Trans on Man-Machine Systems, MMS-10(2) 47-53, (June 1969).

9. B. Noble, "Methods for computing the Moore-Penrose generalized inverse, and related matters," in: Generalized Inverses and Applications, M. Z. Nashed, Ed., pp. 245-301, Academic Press, 1976. (Proceedings of an Advanced Seminar, Sponsored by the Mathematics Research Center, The University of WisconsinMadison, October 8-10, 1973). 
10. C. W. Wampler, "Manipulator inverse kinematic solutions based on vector formulations and damped least-squares methods," IEEE Transactions on Systems, Man, and Cybernetics, SMC-16, 93-101, (January/February 1986).

11. Y. Nakamura and $H$. Hanafusa, "Inverse kinematic solutions with singularity robustness for robot manipulator control," ASME Joumal of Dynamic Systems, Measurement, and Control, 108(3) 163-171, (September 1986).

12. K. Levenberg, "A method for the solution of certain non-linear problems in least squares," Ouarterly of Applied Mathematics, II(2) 164-168, (1944).

13. J. M. Varah, "A practical examination of some numerical methods for linear discrete ill-posed problems," SIAM Review, 21(1) 100-111, (January 1979).

14. A. N. Tihonov, "Solution of incorrectly formulated problems and the regulation method," Soviet Math, 5(4) 1035-1038, (1963).

15. D. W. Marquardt, "Generalized inverses, ridge regression, biased linear estimation, and nonlinear estimation," Technometrics, 12(3) 591-612, (August 1970).

16. C. L. Lawson and R. J. Hanson, Solving Least Squares Problems. Englewood Cliffs, New Jersey, Prentice-Hall, Inc., 1974.

17. T. Yoshikawa, "Analysis and control of robot manipulators with redundancy," in: Robotics Research: The First Intemational Symposium, M. Brady and R. Paul, Eds., MIT Press, 1984, pp. 735-747.

18. C. A. Klein and B. E. Blaho, "Dexterity measures for the design and control of kinematically redundant manipulators," The Intermational Journal of Robotics Research, 6(2) 72-83, (Summer 1987).

19. C. W. Wampler, "Computer methods in manipulator kinematics, dynamics, and control: A comparative study," Ph.D. Thesis, Stanford University, 1985.

20. C. A. Klein and C. H. Huang, "Review of pseudoinverse control for use with kinematically redundant manipulators," IEEE Transactions on Systems, Man, and Cybernetics, SMC-13(3) 245-250, (March/April 1983).

21. A. K. Cline, C. B. Moler, G. W. Stewart, and J. H. Wilkinson, "An estimate for the condition number of a matrix," SIAM Joumal of Numerical Analysis, 16(2), 368-375, (April 1979).

22. J. D. Dixon, "Estimating extremal eigenvalues and condition numbers of matrices," SLAM Joumal of Numerical Analysis, 20(4) 812-814, (August 1983).

23. A. A. Maciejewski, "The analysis and control of robotic manipulators operating at or near kinematically singular configurations," Ph.D. Thesis, The Ohio State University, 1987.

24. S. Twomey, "The application of numerical filtering to the solution of integral equations encountered in indirect sensing measurements," Journal of the Franklin Institute, 279(2) 95-109, (February 1965).

25. R. Mittra and C. Klein, "Stability and convergence of moment methods solutions," in Numerical and Asymptotic Techniques in Electromagnetics, New York, Springer-Verlag, 1975, pp. 129-163. 